\title{
Shear Wave Elastography: Comparing the Accuracy of Ultrasound Scanners Using Calibrated Phantoms in Experiment
}

\author{
DOI: 10.17691/stm2017.9.4.06
}

Received August 7, 2017

D.V. Safonov, MD, DSc, Professor, Department of Radiation Diagnostics, Faculty of Advanced Medical Training";

P.I. Rykhtik, MD, PhD, Head of the Department of Radiology2;

I.V. Shatokhina, MD, Ultrasound Unit of the Department of Radiology2;

S.V. Romanov, MD, PhD, Director';

S.N. Gurbatov, DSc, Professor, Head of the Department of Acoustics;

I.Yu. Demin, PhD, Associate Professor, Department of Acoustics ${ }^{3}$

${ }^{1}$ Nizhny Novgorod State Medical Academy, 10/1 Minin and Pozharsky Square, Nizhny Novgorod, 603005,

Russian Federation;

2Privolzhsky District Medical Center of Federal Medico-Biologic Agency of Russia,

2 Nizhne-Volzhskaya naberezhnaya St., Nizhny Novgorod, 603005, Russian Federation;

${ }^{3}$ Lobachevsky State University of Nizhni Novgorod, 23 Prospekt Gagarina, Nizhny Novgorod, 603950,

Russian Federation

The aim of the study was to compare the accuracy of shear wave elastography performed by ultrasonic scanners from different manufacturers and to develop the conversion coefficients to recalculate the stiffness values obtained with different instruments using a calibrated phantom.

Materials and Methods. A comparative analysis of the accuracy of stiffness measurements by shear wave point elastography was performed using three commercial ultrasonic scanners: Aixplorer (Supersonic Imagine, France), Acuson S2000 (Siemens, Germany), LOGIQ E9 (GE, USA), and the acoustic system Verasonics (Verasonics Inc., USA). The measurements were carried out using the calibrated phantom CIRS Model 049 Elasticity QA Phantom Spherical (Computerized Imaging Reference Systems Company, USA) that contained spherical inclusions of different stiffness.

Results. With all tested scanners, comparable stiffness values with deviations within the measurement error were obtained. For the less stiff spheres of type I and type II, the obtained values were consistent with to the phantom calibration. For the medium-stiff phantom matrix, the measured values were at the lower limit of the calibration range. With the stiff spheres of type III, we found values lower than those predicted by calibration; the deviation was less pronounced in the linear sensor of the LOGIQ E9 scanner. In all sensors, this discrepancy increased along with increasing stiffness of the object. We developed formulas for recalculating the stiffness values for all tested scanners grouped by pairs. For example, for the pair of linear sensors Acuson S2000-Aixplorer, the conversion formulas of the Young's modulus $E$ and the shear wave velocity $V$ are as follows: $E$ (Aixplorer, $\mathrm{kPa}$ ) $=3.11 \cdot V^{2.06}$ (Acuson $\mathrm{S} 2000$, $\left.\mathrm{m} / \mathrm{s}\right)$ and $V($ Acuson $\mathrm{S} 2000, \mathrm{~m} / \mathrm{s})=0.58 \cdot E^{0.48}($ Aixplorer, $\mathrm{kPa})$.

Conclusion. All tested sensors and scanners have a comparable high accuracy of shear wave point elastography. The tested instruments are more accurate when measuring objects with low stiffness; as the stiffness increases, the deviations from the standard values become greater. The formulas proposed for recalculating the stiffness indices allow an accurate comparison between the shear wave elastography results obtained with different scanners. When used with the same object, the measured stiffness values increase in the row: Acuson S2000 $\rightarrow$ Aixplorer $\rightarrow$ LOGIQ E9.

Key words: shear wave shear elastography; ARFI-elastography; elastometry; Young's modulus; shear wave velocity; Aixplorer; Acuson S2000; LOGIQ E9; Verasonics.

Determination of stiffness of various biological objects by means of point shear wave elastography becomes an increasingly common diagnostic modality; this technology is used in most ultrasonic scanners from various manufacturers. The general physical principles of shear wave velocity measurement are detailed in the medical literature [1-4]; however, specific mechanisms for implementing this technology and methods for determining the stiffness are considered commercial secrets, which are not available for public access.

The lack of the relevant information raises a number of questions. How accurately is the tissue stiffness measured and calculated with scanners from different manufacturers? What technology is more accurate? How

For contacts: Dmitry V. Safonov, e-mail: safonovdv@inbox.ru 
significant is the discrepancy between the instruments? In addition, is it necessary to check the instrument accuracy before the measurement or compare the results obtained with different devices based on different modifications of elastography $[5,6]$ ? These and similar questions are increasingly asked by ultrasound doctors; however, no clear answers are yet available. There are single reports [7] on the comparison between various shear wave elastography scanners.

One source of discrepancies between the results obtained with different scanners is the use of different representation parameters. In some instruments (e.g., Siemens), the result is presented as the shear wave velocity expressed in meters per second $(\mathrm{m} / \mathrm{s})$, while in others (Aixplorer) it is recalculated into the Young's modulus $E-$ the conventional physical index characterizing the stiffness (elasticity) of the media and measured in kilopascals $(\mathrm{kPa})$. It is directly proportional to the product of the medium density $\rho$ and the square of the shear wave velocity $V$ :

$$
E=3 \rho V^{2} \text {. }
$$

According to a simplified theory, the density $\rho$ of any soft tissue is constant and equal to $1 \mathrm{~g} / \mathrm{cm}^{3}$, which allows us to not take this index into account. However, this assumption is only relevant for the density of normal soft tissues. In malignant tumors or in fibrotic tissues, the $\rho$ value may be significantly higher than $1 \mathrm{~g} / \mathrm{cm}^{3}$, which may impact the results obtained with different scanners. In such cases, the Young' modulus automatically calculated by the scanner and the tissue stiffness presented in $\mathrm{kPa}$ may contain a considerable measurement error due to an inaccurate $\rho$ value.

To determine the accuracy of elastometry, two components are necessary. Firstly, it is a calibrated phantom for the determination of stiffness in the medium with known elastic characteristics. Secondly, one needs an experimental device with a wide range of settings for high-precision measurements, which can be used as a reference for comparison with other devices. In part, the present experiments based on these two components were run at the Laboratory of Biomedical Technologies, Medical Instrumentation and Acoustic Diagnostics at the Acoustics Department of the Lobachevsky State University of Nizhni Novgorod, where we used an experimental acoustic system with an open architecture (Verasonics) [8]. Another part of the experiments using commercial medical ultrasonic scanners was performed at the Privolzhsky District Medical Center of Federal Medico-Biologic Agency of Russia.

The aim of the study was to compare the accuracy of shear wave elastography performed by ultrasonic scanners from different manufacturers and to develop the conversion coefficients to recalculate the stiffness values obtained with different instruments using a calibrated phantom.

Materials and Methods. Shear wave elastography incorporates two interconnected technologies: point elastography and two-dimensional elastography. With point shear wave elastography (pSWE), shear waves arise due to an exposure to the radiation pressure caused by a strong focused ultrasonic pulse. Another name for this method, ARFI, comes from the abbreviation of "acoustic radiation force impulse" [9].

The principle of point elastography is that at an appropriate depth, a sufficiently high radiation pressure is created by an intense focused short acoustic pulse within a single ultrasonic beam. The energy of this pressure is transferred to the environment, where shear waves are generated and spread from the focus towards the periphery in the plane perpendicular to the scanning axis, just as circles on the water spread from the point where the stone falls $[10,11]$. Then, after the initial pulse, the object (e.g., tissue) is scanned with conventional ultrasonic waves at short time intervals. The resulting signal reflects the displacement of the selected points of the medium from their original position caused by the initial shear wave. The signal also carries the information on the shear wave arrival time. Knowing the distance from the focus to a specific point and the arrival time of the shear wave, we can determine its velocity. The higher the stiffness of the medium, the higher the shear wave velocity. The method of point elastography allows accurate calculating of the tissue stiffness in the limited space where shear waves propagate. Therefore, the term "elastometry" is a legitimate synonym for the designation of this technology.

Further technical development of point elastography was achieved by creating two-dimensional shear wave elastography (2D-SWE) or supersonic shear wave elastography (sSWE). The latter name was given by the Supersonic Imagine Company (France) that first patented the method and implemented it by producing the Aixplorer ultrasonic scanner [12].

In this modification, the focal points of the radiation pressure are sequentially switched through the scanning depth in the zone of interest; each ultrasonic beam generates its own shear waves, similarly to point elastography. The speed of this switching considerably exceeds the speed of the shear waves transverse propagation. As a result, the front lines of shear waves from all focal points sum up into a single front with a conical surface (the Mach cone) propagating perpendicular to the scan axis through the entire depth of the zone of interest.

An ultrasonic scanner measures the shear wave velocity at all depths so to form a two-dimensional color elastogram where sections of different stiffness are encoded in different colors, which allows one to visually assess the elasticity of the area under examination; in addition, point elastography provides the measure of the stiffness index anywhere in the zone of interest. The method makes it possible to obtain qualitative images of the stiffness and to accurately quantify it.

In this study, we used a single calibrated phantom with the known stiffness characteristics to test and compare 


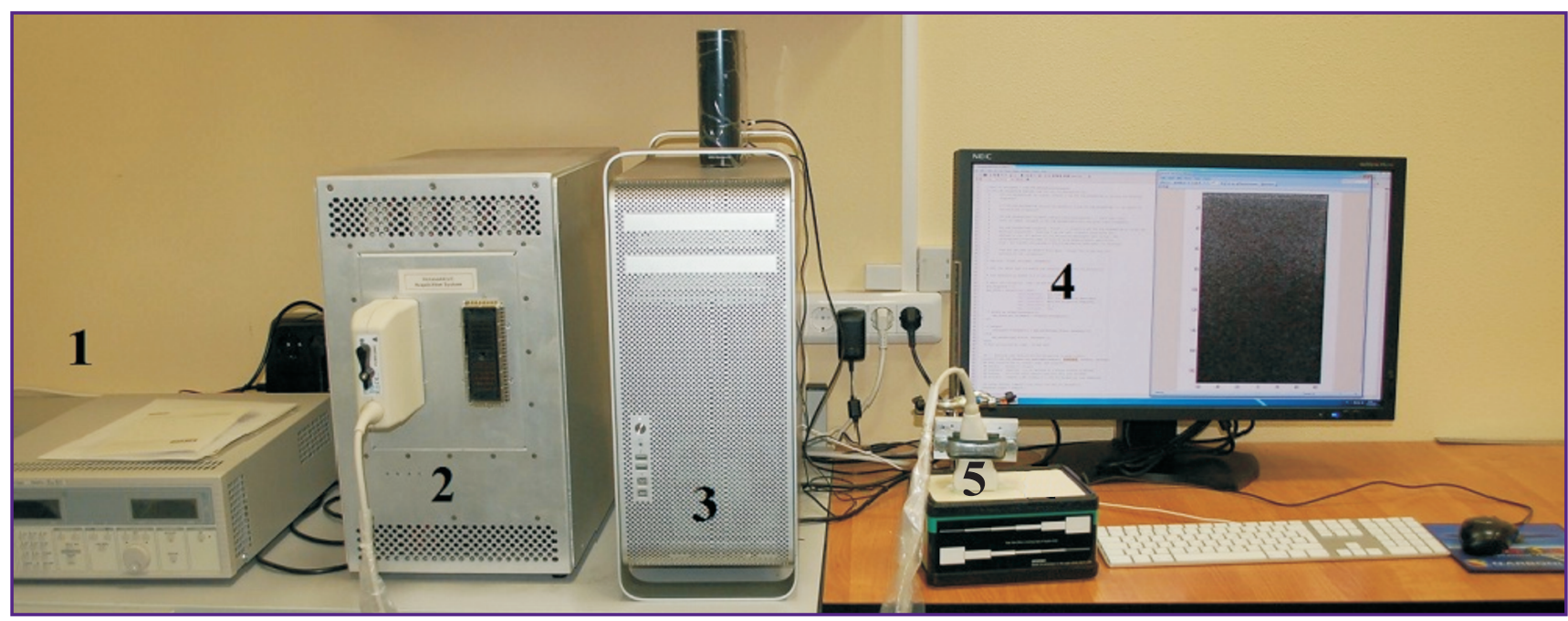

Figure 1. Verasonics acoustic system:

(1) power supply, (2) control unit, (3) computer, (4) monitor, (5) phantom and ultrasonic sensor

four different scanners, as follows: Aixplorer (Supersonic Imagine, France) with convex - XC6-1 (1-6 MHz frequency range), linear - SL15-4 (4-15 MHz) and cavity - SE12-3 (3-12 MHz) multifrequency sensors; Acuson S2000 (Siemens, Germany) with convex 4C1 (1-4.5 MHz) and linear - 9L4 (4-9 MHz) sensors; LOGIQ E9 (GE, USA) with convex - C1-5-D (1.8$5 \mathrm{MHz})$ and linear - 11L-D (4.2-11 MHz) sensors, as well as the Verasonics acoustic system (Verasonics Inc., USA) with an open architecture and the L7-4 linear sensor with a specified operating frequency of $5 \mathrm{MHz}$. The scanners Acuson S2000, LOGIQ E9 and Verasonics are based solely on point elastography, while Aixplorer - explores both point and two-dimensional shear wave elastography.

The Verasonics system is a universal ultrasound device (Figure 1), designed to study the elastic properties of various biological or artificial objects, to optimize algorithms for the processing of acoustic signals and to develop the most informative settings for scanners. The major advantage of the system is its openness, i.e., the ability to change the parameters of ultrasonic waves over a wide range (for example, the number of radiating and receiving channels - from 128 to 256, the carrier wave frequency — from 1 to $15 \mathrm{MHz}$, the power of ultrasound - up to $1,000 \mathrm{~W}$ ) and to program these parameters to meet the tasks and the objectives. The received echo signals are recorded by the instrument and are available for post-processing in the form of arrays of numerical data. The entire scenario of sending pulses, receiving and processing data, as well as constructing an image is programmed by the user in MATLAB.

In the present study, the system was set up according to the standard settings used in commercial ultrasound scanners. Unlike the Verasonics acoustic system, the ability to change the parameters of the generated ultrasound signal is limited to variations in the signal intensity within a safe range, as well as to postprocessing of the received echo signals. The rest of the technical characteristics of the ultrasonic waves are constant and may in part be a commercial secret.

The study was performed using the calibrated polymeric phantom CIRS Model 049 Elasticity QA Phantom Spherical (Computerized Imaging Reference Systems Company, USA), intended to be used as a reference object in measuring the stiffness of various elastic media. The phantom has the form of a rectangular block sized at $20 \times 15 \times 10 \mathrm{~cm}$ that contains a uniform matrix (background) with 2 rows of spherical inclusions of different stiffness, 10 and $20 \mathrm{~mm}$ in diameter located at depths of 15 and $35 \mathrm{~mm}$, respectively (Figure 2).

The phantom is made of the Zerdine polymer with acoustic properties that are not sensitive to fluctuations of ambient temperature or to compression of the sensor, and are as close as possible to the acoustic characteristics of human soft tissues: the ultrasound propagation velocity is $1,540 \pm 10 \mathrm{~m} / \mathrm{s}$, the attenuation coefficient is $0.5 \pm 0.05 \mathrm{~dB} / \mathrm{cm} \cdot \mathrm{MHz}$, and the density is $1.04 \mathrm{~g} / \mathrm{cm}^{3}$. The spherical inclusions inside the phantom have four different degrees of stiffness (I-IV) with known and calibrated values of the Young's modulus, as indicated in the accompanying documentation (Table 1).

We compared the stiffness indices of spherical inclusions of type I-III since none of normal or pathological tissues has the stiffness close to the type IV values.

From the place of its origin, the shear waves propagate in all directions within the plane perpendicular to the scanning axis. To produce a measurable stiffness the waves have to pass a certain distance within the object. This distance should be long enough to measure 


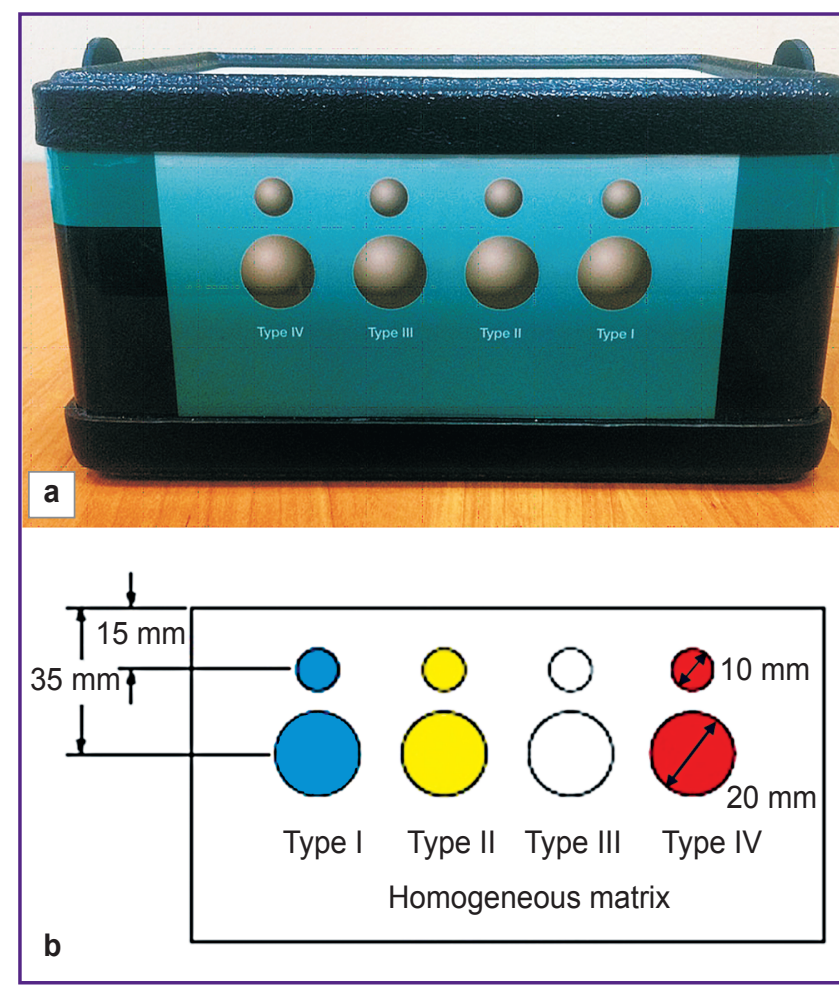

Figure 2. Appearance (a) and location of the spheres inside the polymer phantom (b) CIRS Model 049 Elasticity QA Phantom Spherical (USA)

Table 1

Stiffness of the spherical inclusions: calibration values

\begin{tabular}{ccccc}
\hline \multicolumn{5}{c}{ Young's modulus (kPa) } \\
Type I & Type II & $\begin{array}{c}\text { Homogeneous } \\
\text { matrix } \\
\text { (background) }\end{array}$ & Type III & Type IV \\
\hline $10.0 \pm 4.0$ & $13.0 \pm 4.0$ & $28.0 \pm 7.0$ & $58.0 \pm 10.2$ & $111.0 \pm 18.0$ \\
\hline
\end{tabular}

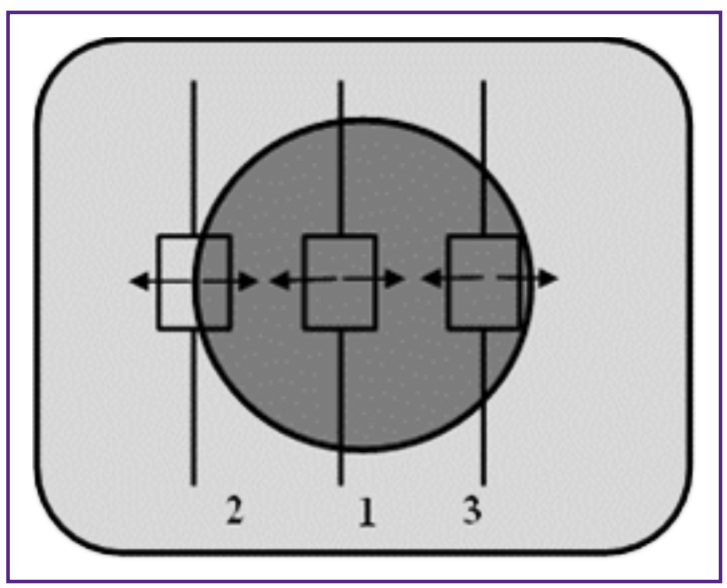

Figure 3. Correct (1) and incorrect $(2,3)$ positions of the control volume in the spherical inclusion during point shear wave elastography measurements

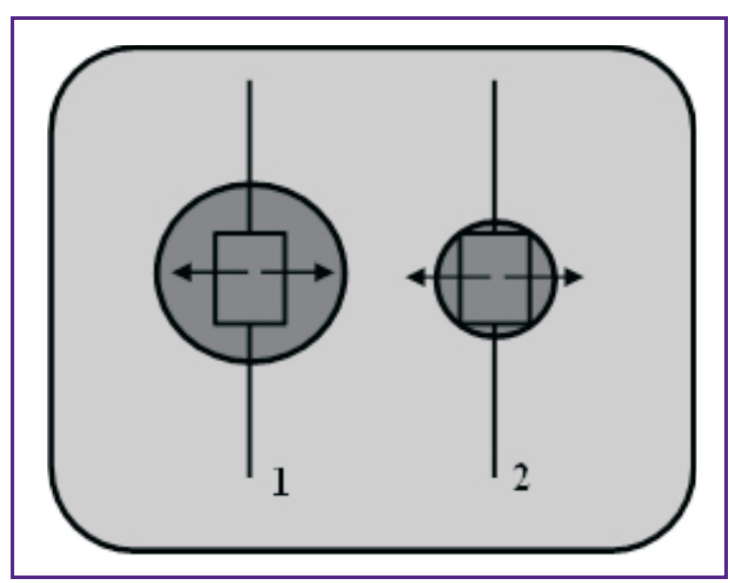

Figure 4. Relationship between the control volume and the spherical inclusion diameter: correct with a diameter of $20 \mathrm{~mm}(1)$ and incorrect with a diameter of $10 \mathrm{~mm} \mathrm{(2)}$
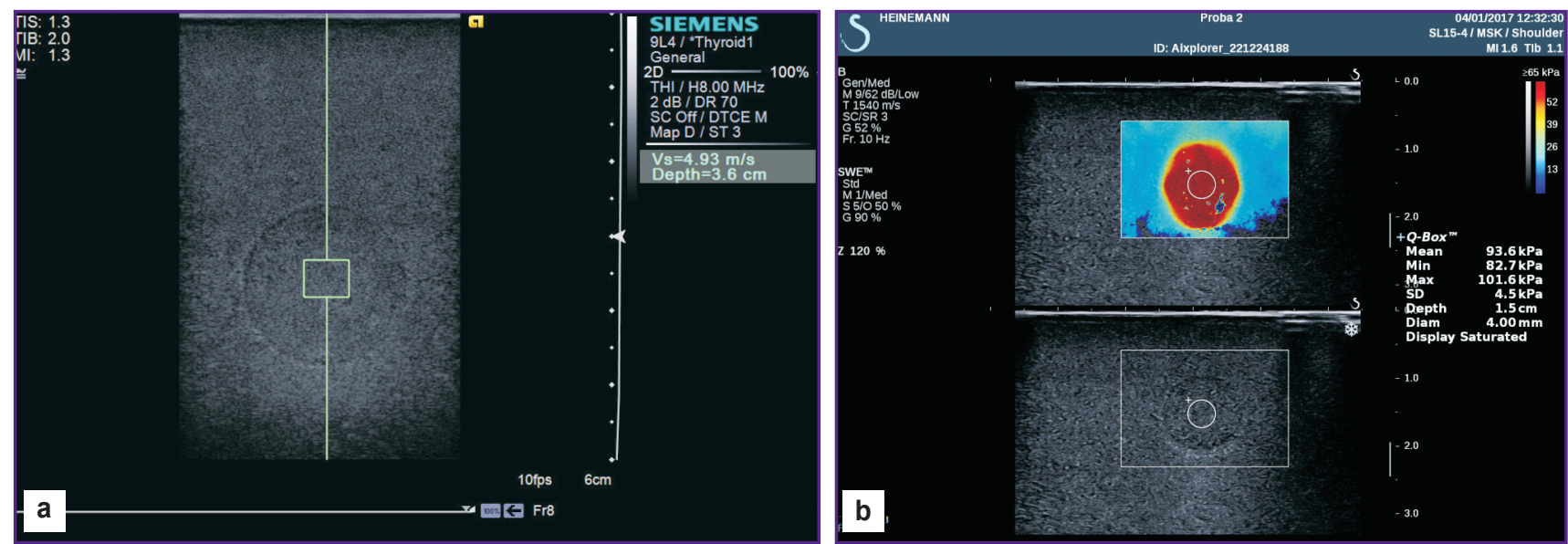

Figure 5. Determination of stiffness of the spherical inclusion using the shear wave velocity with the Acuson S2000 scanner (a) and using the Young's modulus with the Aixplorer scanner (b) 
the shear wave velocity in every direction; otherwise the device may produce some average velocity calculated from the total data obtained in this area (Figure 3 ).

The device is unable to determine whether the wave propagates inside the object or outside it. It calculates and displays the values of the maximum and minimum velocities (Young's modulus) and/or some mean value based on averaging all values obtained within a certain distance (in all directions) from the focus of shear wave generation. This algorithm is installed in the software of all scanners, regardless of the manufacturer, possibly with some technological or mathematical differences concerning the numerical calculations; the latter factor is a commercial secret, not disclosed by the manufacturer.

The above technical features require some adjustment of the reference object and the measurement method. Therefore, for our study, we chose larger spheres of $20 \mathrm{~mm}$ in diameter with the measurement zones located in the central parts of the spheres. These adjustments made it possible to obtain correct values of the shear wave velocity or the Young's modulus precisely inside the sphere without involving the surrounding matrix (Figure 4). With each scanner, we carried 5 measurements of the shear wave velocity in each sphere; as a result the mean values and their standard deviations were determined (Figure 5). The shear wave velocity in the matrix itself was measured at a sufficient distance from the spheres.

Results and Discussion. At the first stage of the study, we measured and compared the stiffness values obtained with the phantom when the convex and linear scanners were used (Table 2).

With all the above convex and linear sensors, comparable stiffness values with differences within the measurement errors were obtained. For spheres of type I and II (low stiffness values), the results were fully consistent with the respective calibration (passport) values. For the medium-stiffness matrix (background) of the phantom, the measured values were at the lower limits of the calibration range. With the high stiffness spheres of type III, the measured values were considerably lower than those obtained in the calibration; these deviations were less pronounced for the linear sensor of the LOGIQ E9 scanner. For all tested sensors, this discrepancy versus the calibration values increased with increasing stiffness of the phantom spheres.

Using the Aixplorer scanner, we compared the accuracy of determining the stiffness of the spheres as measured with the abdominal, linear or cavity sensors (Table 3). For the elastic spheres of type

Table 2

Table 3
I and II and the phantom matrix, all sensors showed practically identical and highly accurate results. In the experiment with the stiff spheres of type III, all sensors showed underestimated values with no differences between the sensors. For the super-stiff spheres of type IV, we found a significant underestimation of the measured values with significant differences between the sensors.

In our opinion, the obtained results can be explained by the fact that the manufacturing companies optimize their elastometry scanners so to accurately measure stiffness within the range similar to that in biological objects; the highest levels of accuracy and reproducibility are achieved for the range of 7.0-33.0 kPa.

At the second stage of the study, we developed the formulas to recalculate the stiffness values obtained with different scanners. The formulas have been derived from the measurement data processed within Excel tables (see Tables 2 and 3); the presented equations are based on the linear and quadratic trend lines and allow determining the quantitative relationships between the readings produced by different ultrasonic scanners. The recalculation factor for the Young's modulus values is a

Stiffness values obtained in elastometry with various devices

\begin{tabular}{|lcccc}
\hline \multirow{2}{*}{$\begin{array}{c}\text { Scanner namel } \\
\text { sensor type }\end{array}$} & Type I & Type II & $\begin{array}{c}\text { Homogeneous } \\
\text { matrix } \\
\text { (background) }\end{array}$ & Type III \\
\cline { 2 - 5 } & \multicolumn{4}{c}{ Convex sensors } \\
\hline Aixplorer/XC6-1 & $8.42 \pm 0.38$ & $11.08 \pm 0.90$ & $23.13 \pm 0.62$ & $32.18 \pm 1.06$ \\
\hline Acuson S2000/4C1 & $7.16 \pm 0.43$ & $11.67 \pm 0.78$ & $20.12 \pm 1.10$ & $29.28 \pm 2.10$ \\
\hline LOGIQ E9/C1-5-D & $9.67 \pm 0.35$ & $11.94 \pm 0.69$ & $22.53 \pm 0.74$ & $35.62 \pm 3.15$ \\
\hline Aixplorer/SL15-4 & $7.05 \pm 0.21$ & $11.20 \pm 0.48$ & $20.59 \pm 0.21$ & $33.19 \pm 1.61$ \\
\hline Acuson S2000/9L4 & $6.66 \pm 0.71$ & $10.11 \pm 0.69$ & $20.56 \pm 0.31$ & $28.35 \pm 2.21$ \\
\hline LOGIQ E9/11L-D & $8.35 \pm 0.89$ & $11.26 \pm 0.54$ & $23.25 \pm 0.25$ & $45.78 \pm 3.85$ \\
\hline Verasonics/L7-4 & $6.38 \pm 0.31$ & $10.24 \pm 0.58$ & $23.15 \pm 0.35$ & $28.12 \pm 2.68$ \\
\hline
\end{tabular}

Stiffness values obtained in elastometry with different sensors of the Aixplorer scanner

\begin{tabular}{|cccccc}
\hline & \multicolumn{5}{c}{ Young's modulus (kPa) } \\
Sensor type & Type I & Type II & $\begin{array}{c}\text { Homogeneous } \\
\text { matrix } \\
\text { (background) }\end{array}$ & Type III & Type IV \\
\hline Convex, XC6-1 & $8.42 \pm 0.38$ & $11.08 \pm 0.90$ & $23.13 \pm 0.62$ & $32.18 \pm 1.06$ & $53.35 \pm 0.91$ \\
\hline Linear, SL15-4 & $7.05 \pm 0.21$ & $11.20 \pm 0.48$ & $20.59 \pm 0.21$ & $33.19 \pm 1.61$ & $61.18 \pm 2.73$ \\
\hline Cavity, SE12-3 & $7.18 \pm 0.22$ & $9.97 \pm 0.57$ & $20.87 \pm 0.67$ & $32.74 \pm 2.65$ & $72.81 \pm 9.31$ \\
\hline
\end{tabular}


linear function, whereas the recalculation for both the Young's modulus $E$ and the shear wave velocity $V$ is quadratic.

In the mathematical terms, the simplest task was a comparison between the results obtained with the linear sensors of the Acuson S2000 scanner and the Verasonics acoustic system, since both instruments measured the shear wave velocity directly:

$$
\begin{gathered}
V(\text { Acuson S2000, m/s })= \\
=1.04 \cdot V(\text { Verasonics }, \mathrm{m} / \mathrm{s})-0.07 ; \\
V(\text { Verasonics }, \mathrm{m} / \mathrm{s})= \\
=0.95 \cdot V(\text { Acuson } \mathrm{S} 2000, \mathrm{~m} / \mathrm{s})+0.09 .
\end{gathered}
$$

As can be seen from the above formulas, the differences in the calculations turned out to be minimal and did not exceed the measurement error; so with the identical settings, the Acuson S2000 can be considered a clinical analogue of the Verasonics device. The obtained data indicate good reproducibility of the measurements and emphasize that the results can be easily compared by direct calculation of shear wave velocity.

The Verasonics acoustic system, used in physical experiments on shear waves in various media, confirmed the high accuracy of the Acuson S2000 clinical scanner. Since this acoustic system is experimental and not used in clinical practice, the conversion formulas for it are of more theoretical value.

For linear sensors of the Acuson S2000 and Aixplorer scanners, the recalculation of the shear wave velocity $V$ and the Young's modulus $E$, based on the quadratic relation of these parameters, is expressed by the following equations:

$$
\begin{aligned}
& V(\text { Acuson S2000, } \mathrm{m} / \mathrm{s})=0.58 \cdot E^{0.48}(\text { Aixplorer, } \mathrm{kPa}) \\
& E(\text { Aixplorer, } \mathrm{kPa})=3.11 \cdot V^{2.06}(\text { Acuson } \mathrm{S} 2000, \mathrm{~m} / \mathrm{s}) \text {. }
\end{aligned}
$$

As we can see, the obtained formula for conversion (5) is close to the classical expression (1) but the proposed corrections allow us to compare the values with a higher accuracy.

For the linear sensors of the Acuson S2000 and LOGIQ E9 scanners, the conversion formulas were as follows:

$$
V\left(\text { Acuson S2000, m/s) }=0.64 \cdot E^{0.42}(\text { LOGIQ E9, kPa); }\right.
$$

$$
\begin{gathered}
E(\text { LOGIQ E9, kPa) }= \\
3.06 \cdot V^{2.28}(\text { Acuson S2000, m/s }) .(7)
\end{gathered}
$$

Graphically, formulas (5) and (7) for the shear wave velocity $V$ (of the Acuson S2000 scanner) conversion into the Young's modulus $E$ (of the Aixplorer and LOGIQ E9 scanners) are shown in Figure 6. If, however, the shear wave velocity (obtained with the Acuson S2000 scanner) is first converted into the Young's modulus by formula (1), then it can be converted to the Young's moduli (for the linear sensors of the Aixplorer and LOGIQ E9 scanners) using the formulas presented in Table 4. These relationships can also be represented graphically: Figure 7 shows the conversion graphs of the Young's moduli (of the LOGIQ E9 scanner) to the corresponding index (of the Aixplorer and Acuson S2000 scanners).

As it follows from Table 4, the direct and inverse formulas in the pairs Acuson S2000-Aixplorer and Verasonics-Aixplorer, as well as in the pairs Acuson S2000-LOGIQ E9 and Verasonics-LOGIQ E9 are very similar, since the recalculation into the Young's modulus is based on the shear wave velocities measured with instruments, similar in their technical implementation of elastometry. 
The conversion formulas for the Aixplorer-LOGIQ E9 pair have a slightly different numerical expression since both scanners first display the readings in kilopascals of the Young's modulus.

Similarly, based on the data in Table 2 we have determined formulas for the conversion of the Young's moduli applicable to the convex sensors (Table 5).

Experimental measurements using a calibrated phantom and calculating the conversion formulas can be performed with different ultrasonic scanners adapted for point shear wave elastography. As a result one can create a unified table for the recalculation of the Young's modulus values (or the shear wave velocity values) for all medical ultrasound scanners designed for point elastography. The necessity and importance of this procedure are undeniable since elastography has a recognized diagnostic value and has been increasingly used in clinical practice. We evidence a greater number of operating scanners in clinics as well as a greater

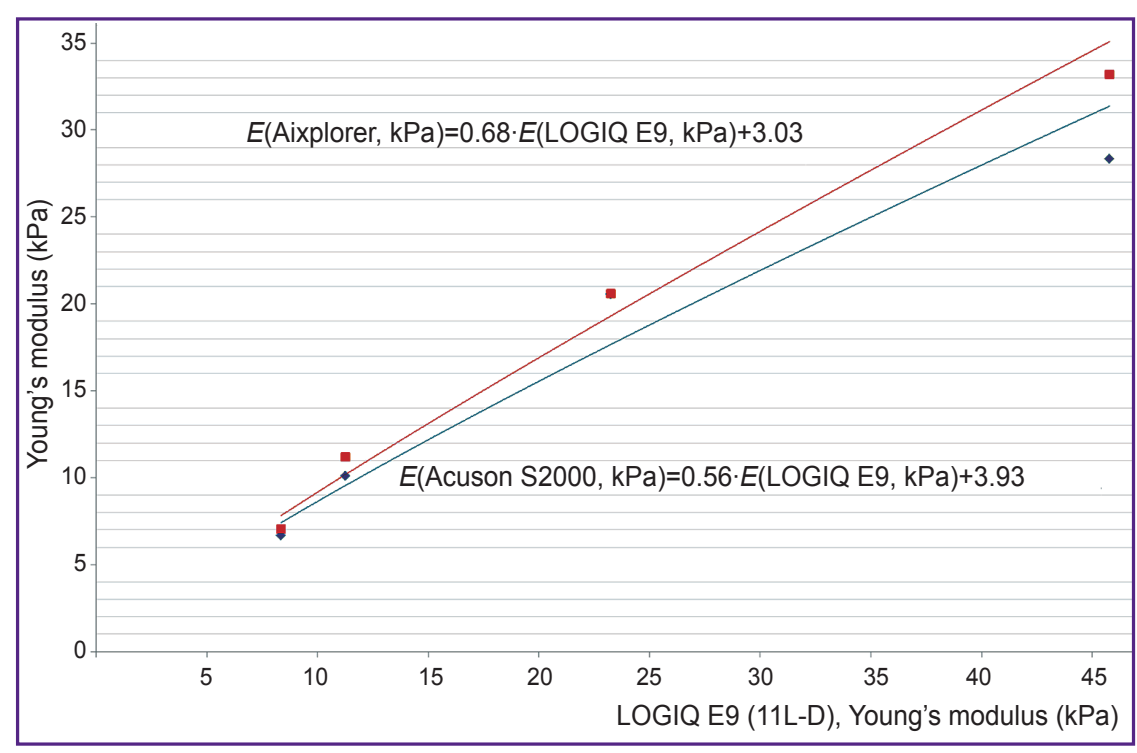

Figure 7. Graphic representation of the formulas for converting the Young's moduli from the LOGIQ E9 scanner to the Aixplorer and Acuson S2000 scanners

Table 5

Formulas for converting the Young's moduli in convex sensors

\begin{tabular}{|c|c|}
\hline Direct formula (kPa) & Inverse formula (kPa) \\
\hline$E($ Acuson $S 2000)=0.88 \cdot E$ (Aixplorer) +0.61 & $E($ Aixplorer $)=1.12 \cdot E($ Acuson $S 2000)-0.47$ \\
\hline$E($ Acuson S2000) $=0.82 \cdot E($ LOGIQ E9) +0.79 & $E($ LOGIQ E9) $=1.21 \cdot E($ Acuson S2000) -0.61 \\
\hline$E($ Aixplorer $)=0.92 \cdot E($ LOGIQ E9) +0.31 & $E($ LOGIQ E9) $=1.07 \cdot E($ Aixplorer $)$ \\
\hline
\end{tabular}
number of their manufacturers.

Despite the uniform physical basis

of elastometry, the technical implementations of this technique vary from a manufacturer to a manufacturer, which inevitably leads to discrepancies between the results obtained with different scanners and necessitates the use of recalculation formulas.

The difference between the corrected numerical values and the initial ones can be significant and can impact the diagnostic results for liver fibrosis as expressed according to the Metavir score. This notion can easily be verified by entering different numerical values of the Young's modulus in the recalculation formulas, Tables 4 and 5, and calculating the stiffness values for different scanners.

We found that the measurement deviations increased with increasing stiffness, which, in the case of the liver, could produce overestimated values of the liver fibrosis. Based on the primary measurements (see Table 2) and the conversion formulas (see Tables 4 and 5), we propose the following sequence of scanners according to the increasing values of stiffness measured in the same object: Acuson S2000 $\rightarrow$ Aixplorer $\rightarrow$ LOGIQ E9.

Conclusion. The tested scanners Aixplorer, Acuson S2000, LOGIQ E9 and the experimental acoustic system Verasonics have a comparably high accuracy of shear wave point elastography as applied to stiffness measurements. The signal frequency and the shape of the sensors do not make differences in the sensor accuracy. All tested sensors and scanners are oriented to measuring objects with low stiffness values; there the greatest accuracy of measurements is achieved. As the stiffness increases, deviations from the standard values also increase. The formulas developed here for recalculation of the stiffness values allow one to correctly compare the shear wave elastography data obtained with different scanners.

Financial Support. The study was supported by the Russian Foundation for Basic Research (grant 15-4202586).

Conflicts of Interest. The authors have no conflicts of interest.

\section{References}

1. Rudenko O.V., Safonov D.V., Rykhtik P.I., Gurbatov S.N., Romanov S.V. Physical bases of elastography. Part 2. Shear wave elastography (Lecture). Radiologiya Praktika 2014; 4(46): 62-72.

2. Bamber J., Cosgrove D., Dietrich C.F., Fromageau J., Bojunga J., Calliada F., Cantisani V., Correas J.M., D'Onofrio M., Drakonaki E.E., Fink M., Friedrich-Rust M., Gilja O.H., Havre R.F., Jenssen C., Klauser A.S., Ohlinger R., Saftoiu A., Schaefer F., Sporea I., Piscaglia F. EFSUMB guidelines and recommendations on the clinical use of 
ultrasound elastography. Part 1: Basic principles and technology. Ultraschall Med 2013; 34(2): 169-184, https://doi. org/10.1055/s-0033-1335205.

3. Mitkov V.V., Mitkova M.D. Ultrasound shear wave elastography. Ul'trazvukovaya $i$ funktsional'naya diagnostika 2015; 2: 94-108.

4. Elastografiya sdvigovoy volny: analiz klinicheskikh primerov [Shear wave elastography: an analysis of clinical examples]. Pod red. Borsukova A.V. Smolensk: Smolenskaya gorodskaya tipografiya; 2017.

5. Morozova T.G., Borsukov A.V. Clinical relevance of compression elastography in differential diagnosis of pancreatic cystic masses. Sovremennye tehnologii v medicine 2014; 6(2): 103-107.

6. Surkov A.N., Namazova-Baranova L.S., Vashakmadze N.D., Gevorkjan A.K., Tomilova A.Ju., Potapov A.S., Simonova O.I., Kuzenkova L.M., Baranov A.A. Transient elastography is a noninvasive method to diagnose hepatic fibrosis stages in children with rare diseases. Sovremennye tehnologii v medicine 2016; 8(3): 56-63, https:// doi.org/10.17691/stm2016.8.3.06.

7. Mulabecirovic A., Vesterhus M., Gilja O.H., Havre R.F. In vitro comparison of five different elastography systems for clinical applications, using strain and shearwave technology.
Ultrasound Med Biol 2016; 42(11): 2572-2588, https://doi. org/10.1016/j.ultrasmedbio.2016.07.002.

8. Khalitov R.S., Gurbatov S.N., Demin I.Y. The use of the Verasonics ultrasound system to measure shear wave velocities in CIRS phantoms. Physics of Wave Phenomena 2016; 24(1): 73-76, https://doi.org/10.3103/s1541308x16010143.

9. Sarvazyan A.P., Rudenko O.V., Swanson S.D., Fowlkes J.B., Emelianov S.Y. Shear wave elasticity imaging: a new ultrasonic technology of medical diagnostics. Ultrasound Med Biol 1998; 24(9): 1419-1435, https://doi.org/10.1016/ s0301-5629(98)00110-0.

10. Ophir J., Céspedes I., Ponnekanti H., Yazdi Y., Li X. Elastography: a quantitative method for imaging the elasticity of biological tissues. Ultrason Imaging 1991; 13(2): 111-134, https:/doi.org/10.1016/0161-7346(91)90079-w.

11. Palmeri M.L., Frinkley K.D., Zhai L., Gottfried M., Bentley R.C., Ludwig K., Nightingale K.R. Acoustic radiation force impulse (ARFI) imaging of the gastrointestinal tract. Ultrason Imaging 2005; 27(2): 75-88, https://doi.org/10.1177/0 16173460502700202.

12. Bercoff J., Tanter M., Fink M. Supersonic shear imaging: a new technique for soft tissue elasticity mapping. IEEE Trans Ultrason Ferroelectr Freq Control 2004; 51(4): 396-409, https://doi.org/10.1109/tuffc.2004.1295425. 